from CareRA baseline of DAS28-CRP and HAQ was assessed via linear mixed models. All adverse events (AEs), considered to be clinically relevant by investigators, and DMARD/GCs therapy were registered.

Results: Of 322 eligible patients, $252(78 \%)$ were included in CareRA-plus, of which $203(81 \%)$ completed the study. Characteristics and outcomes at the CareRA closing visit (year 2) did not differ between patients entering CareRA-plus or not. DAS28-CRP<2.6 at year 5 in high-risk patients was $72 \%, 77 \%$ and $64 \%$ in the Classic, Slim and Avant-Garde group respectively $(p=0.403)$. In the Iongitudinal analyses, all treatment arms in the high-risk group had comparable DAS28-CRP $(p=0.921)$ and HAQ scores over time $(p=0.540)$. In the low-risk population, $83 \%$ of patients in the Slim and $82 \%$ in the TSU arm had DAS28CRP $<2.6$ at year $5(p=0.945)$. Low-risk patients starting Cobra-Slim had lower DAS28-CRP scores over 5 years than those receiving TSU $(p=0.002)$. HAQ score over time did not differ $(p=0.129)$. In high-risk patients, the total numbers of AEs throughout CareRA-plus, were 70 in 36 Classic, 95 in 48 Slim and 80 in 36 Avant-Garde patients $(p=0.182)$. In the low-risk group there were 18 AEs in 10 Slim and 36 in 17 TSU patients $(p=0.048)$. During the 5 -year study, biologics were initiated in $22 \%$ of all patients: $23 \%$ of Classic, $23 \%$ of Slim high-risk, $25 \%$ of Avant-Garde, $17 \%$ of Slim low-risk, and $15 \%$ of TSU patients. At the year 5 visit, $71 \%, 61 \%$ and $50 \%$ of high-risk patients were on csDMARD monotherapy (mostly MTX) in Classic, Slim and Avant-Garde respectively. Of the low-risk group, $65 \%$ in COBRA-Slim and $62 \%$ in TSU were taking a single csDMARD. At the year 5 visit, $9 \%$ of all participants received chronic oral GC therapy (>3 months).

Conclusion: All intensive treatment strategies resulted in excellent long-term clinical outcomes. Initial Cobra Slim therapy showed comparable 5-year effectiveness as Cobra Classic and Avant-Garde in high-risk early RA patients and better efficacy and safety than conservative step up treatment in low-risk patients.
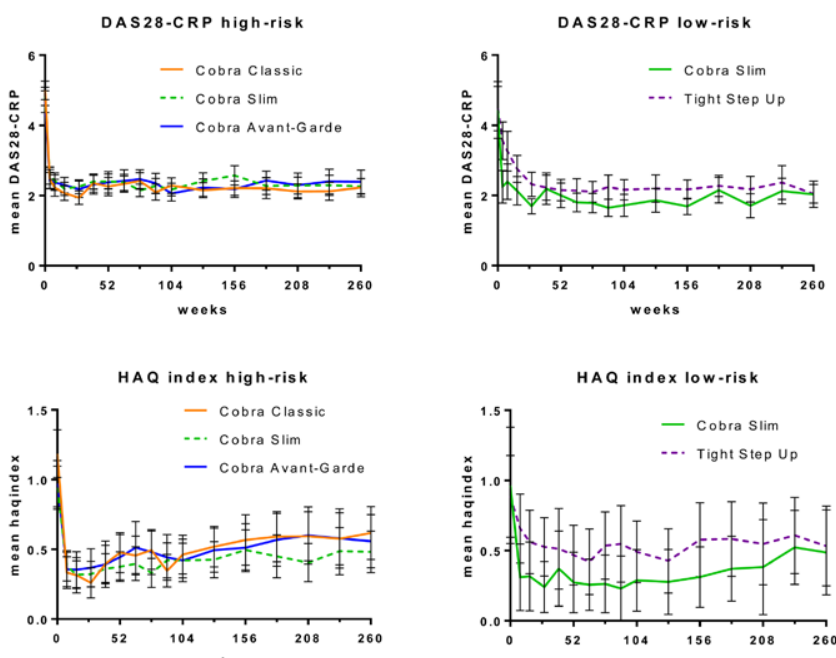

Figure 1. Mean disease activity by DAS28-CRP or mean functionality by HAQ index scores for high-risk or low-risk patients.

References:

[1] Stouten, V. et al. Effectiveness of different combinations of DMARDs and glucocorticoid bridging in early rheumatoid arthritis: two-year results of CareRA. Rheumatology (Oxford). (2019) doi:10.1093/rheumatology/kez213.

Disclosure of Interests: : Veerle Stouten: None declared, Rene Westhovens Grant/research support from: Celltrion Inc, Galapagos, Gilead, Consultant of: Celltrion Inc, Galapagos, Gilead, Speakers bureau: Celltrion Inc, Galapagos, Gilead, Diederik De Cock: None declared, Sofia Pazmino: None declared, Johan Joly: None declared, Delphine Bertrand: None declared, Kristien Van der Elst: None declared, Patrick Verschueren Grant/research support from: Pfizer unrestricted chair of early RA research, Speakers bureau: various companies DOI: 10.1136/annrheumdis-2020-eular.2013

\section{THU0215 WHOLE BLOOD TRANSCRIPTIONAL CHANGES FOLLOWING SELECTIVE INHIBITION OF JANUS KINASE 1 (JAK1) BY FILGOTINIB IN ADULTS WITH MODERATELY-TO-SEVERELY ACTIVE RHEUMATOID ARTHRITIS WITH PRIOR INADEQUATE RESPONSE TO METHOTREXATE (FINCH1)}

P. C. Taylor ${ }^{1}$, B. Downie ${ }^{2}$, E. Elboudwarej ${ }^{2}$, S. Kim², A. Hertz ${ }^{2}$, A. M. Mirza², J. Siegel ${ }^{2}$, R. E. Hawtin ${ }^{2}$, J. Liu' ${ }^{2}{ }^{1}$ University of Oxford, Botnar Research Centre,
Nuffield Dept Orthopaedics, Rheumatology and Musculoskeletal Sciences, Oxford, United Kingdom; ${ }^{2}$ Gilead Sciences, Inc., Foster City, United States of America

Background: Filgotinib (FIL), an oral selective JAK1 inhibitor, has shown efficacy and safety in multiple phase 3 studies in adults with moderately-to-severely active rheumatoid arthritis (RA). We have previously described the molecular response to FIL in large-scale RNA sequencing studies of gene expression in other RA populations ${ }^{1-3}$ and herein conducted a similar study in RA patients (pts) with prior inadequate response to methotrexate (MTX; FINCH1).

Objectives: Identify RA-associated gene transcripts and biological pathways that are altered in response to FIL treatment.

Methods: RA pts who had an inadequate response to MTX were enrolled in FINCH1 (ClinicalTrials.gov NCT02889796) and randomized to receive either a stable dose of MTX with placebo (PBO+MTX), adalimumab (ADA+MTX), or one of two doses of FIL (FIL 100mg+MTX, FIL 200mg+MTX) once daily (QD). Whole blood samples were collected from pts using PAXgene tubes at baseline, week 4, and week 12. RNA from these samples was extracted and sequenced on the Illumina HiSeq 2500 platform following globin RNA depletion. Correlations between baseline gene expression and disease measurements were performed using Spearman's rank partial correlation with covariates. Differentially expressed genes (DEGs) were identified using voom-limma. Pathway analysis was performed on v6.1 of the Molecular Signature Database using single sample gene set enrichment analysis (GSEA) with the focus on immune signaling pathways from the Kyoto Encyclopedia of Genes and Genomes (KEGG). A false-discovery rate of $5 \%$ was applied for all analyses.

Results: Differential gene expression analyses comparing baseline samples with after-treatment samples revealed more rapid transcriptional kinetics for FIL-treated pts compared to ADA+MTX-treated pts. No significant DEGs were observed in PBO-treated pts. More significant DEGs were observed in the FIL $200 \mathrm{mg}+\mathrm{MTX}$ arm compared to the FIL 100mg+MTX arm, consistent with the superior clinical efficacy of the FIL $200 \mathrm{mg}$ dosage. As with other FIL clinical trial RNA-seq studies and consistent with the selective MoA of FIL, JAK-STAT pathway-induced genes SOCS2 and CISH were significantly downregulated across both FIL treatment arms and timepoints, but not in the ADA+MTX arm. RA disease activity associated genes ${ }^{2-3}$ FAM20A and METTL7B were significantly reduced at both 4 and 12 weeks only in the FIL 200mg+MTX arm. While no significant changes in KEGG immune signaling pathways were observed in the PBO+MTX arm, a dose-dependent effect on pathway modulation was observed in the FIL arms. The most prominently down-regulated KEGG pathways included JAK-STAT signaling and leukocyte transendothelial migration.

Conclusion: More rapid and sustained changes of transcriptional activity were observed in the whole blood transcriptional profile of RA pts following FIL $200 \mathrm{mg}+\mathrm{MTX}$ compared to ADA+MTX treatment. Dose-dependent changes were observed in FIL-treated pts, most notably in the KEGG JAK-STAT signaling pathway. These observations confirm an inhibition of JAK-STAT signaling by FIL and are consistent with the observed clinical efficacy of FIL in these pts.

\section{References:}

[1] Taylor PC, et al. (EULAR 2018). http://dx.doi.org/10.1136/annrheumdis-2018eular.3759

[2] Taylor PC, et al. (ACR 2018). https://doi.org/10.1093/rheumatology/ kez105.001

[3] Taylor PC, et al. (EULAR 2019). http://dx.doi.org/10.1136/annrheumdis-2019eular.2509

Acknowledgments : This study was funded by Gilead Sciences, Inc. Editoria support was provided by Fishawack Communications Inc and funded by Gilead Sciences, Inc.

Disclosure of Interests: : Peter C. Taylor Grant/research support from: Celgene, Eli Lilly and Company, Galapagos, and Gilead, Consultant of: AbbVie, Biogen, Eli Lilly and Company, Fresenius, Galapagos, Gilead, GlaxoSmithKline, Janssen, Nordic Pharma, Pfizer Roche, and UCB, Bryan Downie Shareholder of: Gilead Sciences Inc., Employee of: Gilead Sciences Inc., Emon Elboudwarej Shareholder of: Gilead Sciences Inc., Employee of: Gilead Sciences Inc., Sam Kim Shareholder of: Gilead Sciences Inc., Employee of: Gilead Sciences Inc., Angie Hertz Shareholder of: Gilead Sciences Inc, Employee of: Gilead Sciences Inc, Amer M. Mirza Shareholder of: Gilead Sciences Inc., Employee of: Gilead Sciences Inc., Jeffrey Siegel Shareholder of: Gilead Sciences Inc., Employee of: Gilead Sciences Inc., Rachael E. Hawtin Shareholder of: Gilead Sciences Inc., Employee of: Gilead Sciences Inc., Jinfeng Liu Shareholder of: Gilead Sciences Inc., Roche, Employee of: Gilead Sciences Inc. DOI: 10.1136/annrheumdis-2020-eular.2996

\begin{tabular}{l|l}
\hline THU0216 & PERIPHERAL PROTEIN BIOMARKER CHANGES \\
& FOLLOWING SELECTIVE INHIBITION OF JANUS \\
& KINASE 1 (JAK1) BY FILGOTINIB IN METHOTREXATE \\
& NAÏVE ADULTS WITH MODERATELY-TO-SEVERELY \\
& ACTIVE RHEUMATOID ARTHRITIS (FINCH3)
\end{tabular}

P. C. Taylor ${ }^{1}$, A. M. Mirza², B. Downie ${ }^{2}$, J. Liu², R. E. Hawtin², E. Elboudwarej². ${ }^{1}$ University of Oxford, Botnar Research Centre, Nuffield Department of 
Orthopaedics, Rheumatology and Musculoskeletal Sciences, Oxford, United Kingdom; ${ }^{2}$ Gilead Sciences Inc., Foster City, United States of America

Background: Filgotinib (FIL), an oral selective JAK1 inhibitor, has shown efficacy and safety in multiple phase 3 studies in adults with moderately-to-severely active rheumatoid arthritis (RA), including those who are naïve to methotrexate (MTX) therapy (FINCH3; NCT02886728).

Objectives: A longitudinal study of protein biomarkers related to JAK signaling $^{1}$, bone biology ${ }^{2}$, immune cell migration ${ }^{2}$, and inflammation ${ }^{2}$ was conducted in FINCH3 pts to identify disease relevant biomarkers that are altered by FIL vs MTX.

Methods: MTX-naive RA pts enrolled in FINCH3 received a stable dose of MTX (MTX mono), FIL200mg monotherapy (FIL200mg mono) or one of two doses of FIL once daily with MTX (FIL100mg+MTX, FIL200mg+MTX). Up to 27 disease relevant biomarkers were evaluated. Baseline $(\mathrm{BL})$ correlation between biomarkers and clinical response measures were analyzed by Spearman Rank. Multiscale bootstrap resampling was used to evaluate significant intra-cluster biomarker membership. Mean changes in biomarker levels from BL to wks 4 , 12 and 24 were compared between arms using MTX-adjusted estimates from a linear mixed effects model, adjusted for age, sex, race and BL biomarker level. A false discovery rate of $5 \%$ was applied for all analyses.

Results: At BL, distinct clusters (CL) of biomarkers differentiated by JAK signaling were identified. The strongest intra-group correlations were in biomarkers upstream of JAK2 signaling (CL1; Rho range 0.88-0.98) and cytokines associated with JAK1 signaling (CL2; Rho range 0.72-0.77). Within MTX-naïve RA pts, there were significant BL correlations between 15 biomarkers and clinical measures. The strongest associations observed were between DAS28CRP and IL6, CXCL10, TNFRI, YKL-40, and CXCL13 (Rho >0.3)

Relative to MTX mono, 23 biomarkers exhibited significant early responses to treatment (any arm, wk 4). The strongest treatment effect observed at wk 4 was a reduction by FIL+MTX (regardless of dose) and FIL200mg mono for CXCL13 (FIL100mg+MTX: $-28.2 \%$; FIL200mg+MTX: -40\%; FIL200mg: -34\%). This reduction was sustained in each arm through 24 wks, with the greatest reduction by FIL200mg+MTX (-37.8\%). Dose differences were observed relative to FIL100mg+MTX, where FIL200mg+MTX led to an early (wk 4) and significantly greater reduction of 9 biomarkers. There was a significant dose difference as a delayed response (wk 24) with a greater reduction by FIL200mg+MTX for 8 biomarkers.

FIL200mg mono produced a greater effect on 18 biomarkers vs MTX mono, remaining significant through wk 24. The greatest effect in FIL200mg mono were reductions by wk 24 in CTX1 (-29.1\%), CXCL13 (-33.2\%), and IL6 (-29.5\%), all of which were biomarkers associated with DAS28CRP at BL. Effects observed at any time point were largely similar between FIL200mg as a mono or in combination with MTX. Four biomarkers were uniquely different between FIL200mg mono and FIL200mg+MTX arms by wk 24: greater increase of MMP7 and decrease of GMCSF in FIL200mg+MTX; greater decrease of TRACP5B and ICAM1 in FIL200mg alone. Conclusion: Treatment through 24 weeks with FIL200mg (mono or with MTX) reduced many of the disease-relevant biomarkers tested; markers related to JAK signaling ${ }^{1}$, bone biology ${ }^{2}$, inflammation ${ }^{2}$, and immune cell migration ${ }^{2}$ in the MTXnaïve RA setting. Changes were significantly reduced relative to MTX mono at wk 4, supporting the rapid onset of FIL clinical efficacy. The current study identified significant reductions of RA-associated disease markers that were unique to FIL mono, supporting the FIL mechanisms of action in the treatment of RA.

References:

[1] Majoros A, et al. Front Immunol. 2017;8:29

[2] Brennan F, and Mclnnes I. J Clin Invest. 2008;118:3537-45

Acknowledgments : This study was funded by Gilead Sciences, Inc. Editorial support was provided by Fishawack Communications Inc and funded by Gilead Sciences, Inc.

Disclosure of Interests: : Peter C. Taylor Grant/research support from: Celgene, Eli Lilly and Company, Galapagos, and Gilead, Consultant of: AbbVie, Biogen, Eli Lilly and Company, Fresenius, Galapagos, Gilead, GlaxoSmithKline, Janssen, Nordic Pharma, Pfizer Roche, and UCB, Amer M. Mirza Shareholder of: Gilead Sciences Inc., Employee of: Gilead Sciences Inc., Bryan Downie Shareholder of: Gilead Sciences Inc., Employee of: Gilead Sciences Inc., Jinfeng Liu Shareholder of: Gilead Sciences Inc., Roche, Employee of: Gilead Sciences Inc., Rachael E. Hawtin Shareholder of: Gilead Sciences Inc., Employee of: Gilead Sciences Inc., Emon Elboudwarej Shareholder of: Gilead Sciences Inc., Employee of: Gilead Sciences Inc.

DOI: 10.1136/annrheumdis-2020-eular.4725

\section{THU0217 \\ UPADACITINIB MONOTHERAPY IN METHOTREXATE- NAÏVE PATIENTS WITH RHEUMATOID ARTHRITIS: RESULTS AT 72 WEEKS FROM SELECT-EARLY}

R. Van Vollenhoven ${ }^{4}$, T. Takeuchi' ${ }^{2}$, M. Rischmueller ${ }^{3}$, R. Blanco ${ }^{4}$, R. Xavier ${ }^{5}$, M. Howard ${ }^{6}$, A. Friedman ${ }^{6}$, Y. Song ${ }^{6}$, V. Strand ${ }^{7} .{ }^{1}$ Amsterdam University Medical Centers, Amsterdam, Netherlands; ${ }^{2}$ Keio Univ School of Medicine, Tokyo, Japan; ${ }^{3}$ The Queen Elizabeth Hospital and University of Adelaide, Adelaide,
Australia; ${ }^{4}$ Hospital Universitario Marques de Valdecilla, Cantabria, Spain; ${ }^{5}$ Universidade Federal do Rio Grande do Sul, Porto Alegre, Brazil; ${ }^{6}$ AbbVie, North Chicago, United States of America; ${ }^{7}$ Stanford University, Palo Alto, United States of America

Background: Upadacitinib (UPA), an oral JAK inhibitor, demonstrated significant improvements in signs, symptoms, and structural inhibition as monotherapy vs methotrexate (MTX) in a randomized, controlled trial (RCT) of MTX-naive RA patients (pts) through 48 weeks (wks). ${ }^{1}$

Objectives: To present the safety and effectiveness of UPA through 72 wks in an ongoing long-term extension (LTE) of the SELECT-EARLY RCT.

Methods: SELECT-EARLY included 2 study periods: (1) a 48-wk double-blind, active comparator-controlled, with pts randomized to UPA monotherapy 15 or $30 \mathrm{mg}$ once daily or MTX (titrated to $20 \mathrm{mg} / \mathrm{wk}$ by Wk8); (2) an LTE, up to 4 years Pts received open-label treatment once the last pt reached Wk48. Rescue therapy was added (MTX, for UPA groups; UPA, for MTX group) to pts not achieving CDAl remission ( $\leq 2.8)$ at Wk26. Non-responder imputation (NRI) was used for missing data as well as for pts receiving rescue therapy. Treatment-emergent adverse events (TEAEs) are summarized per $100 \mathrm{pt}$ yrs (PY) through the cut-off date of 21 Feb 2019, when all pts had reached Wk72. Data are censored at the time of MTX or UPA addition among rescued patients.

Results: Of 945 pts randomized and treated, $781(83 \%)$ completed Period 1. Of these, 775 entered the LTE, including 57 rescued pts (MTX, 33; UPA $15 \mathrm{mg}, 17$; UPA $30 \mathrm{mg}, 7)$. A total of $52(7 \%)$ pts discontinued during the LTE through the cutoff date (primary reasons: AEs [ $n=16,2.1 \%]$; consent withdrawal $[n=12,1.5 \%]$; lost to follow-up [n=10,1.3\%]). Cumulative exposures to monotherapy with MTX UPA $15 \mathrm{mg}$, and UPA $30 \mathrm{mg}$ were $350.6,389.5$, and $383.9 \mathrm{PYs}$, respectively. Both UPA $15 \mathrm{mg}$ and $30 \mathrm{mg}$ as monotherapy was associated with continued statistically significant improvements in disease activity measures vs MTX monotherapy through $72 \mathrm{wks}$ (Table). The safety profiles of the UPA 15 and $30 \mathrm{mg}$ groups were comparable for total TEAEs and numerically higher than MTX. Serious TEAEs and TEAEs leading to discontinuation of study drug were comparable across all groups (Figure). Most AEs of special interest were comparable across MTX and UPA groups, with the exception of higher rates of herpes zoster, opportunistic infections, and elevated creatine phosphokinase among the UPA groups. Two pts receiving MTX monotherapy experienced a venous thromboembolic event, with one event reported on UPA $30 \mathrm{mg}$ and none on UPA $15 \mathrm{mg}$. There were 12 deaths (including 3 non-treatment-emergent) due to varied causes.

Table. Proportion of Patients at Week 72 (NRI)

\begin{tabular}{|c|c|c|c|}
\hline Parameter (\%) & $\begin{array}{l}\text { MTX } \\
\text { Monotherapy }\end{array}$ & $\begin{array}{l}\text { UPA } 15 \mathrm{mg} \text { QD } \\
\text { Monotherapy }\end{array}$ & $\begin{array}{l}\text { UPA } 30 \mathrm{mg} \text { QD } \\
\text { Monotherapy }\end{array}$ \\
\hline ACR20/50/70 & $50 / 39 / 26$ & $71^{\star \star \star} / 62^{\star \star \star} / 47^{\star \star \star}$ & $72^{\star \star \star} / 67^{\star \star \star} / 54^{\star \star \star}$ \\
\hline DAS28(CRP) $\leq 3.2 /<2.6$ & $38 / 28$ & $63^{\star \star \star} / 52^{\star \star \star}$ & $69^{\star \star \star} / 61^{\star \star \star}$ \\
\hline $\mathrm{CDAl} \leq 10 / \leq 2.8$ & $42 / 19$ & $60^{\star \star \star} / 35^{\star \star \star}$ & $69^{\star \star \star} / 44^{\star \star *}$ \\
\hline Boolean Remission & 13 & $29^{\star \star \star}$ & $33^{\star \star \star}$ \\
\hline
\end{tabular}

***, $P<0.001$ for differences between MTX and UPA 15 and UPA $30 \mathrm{mg}$ groups.

MTX, methotrexate; UPA, upadacitinib; QD, once daily; ACR, American College of Rheumatology; DAS28(CRP), 28-joint disease activity index based on C-reactive protein; CDAl, clinical disease activity index.

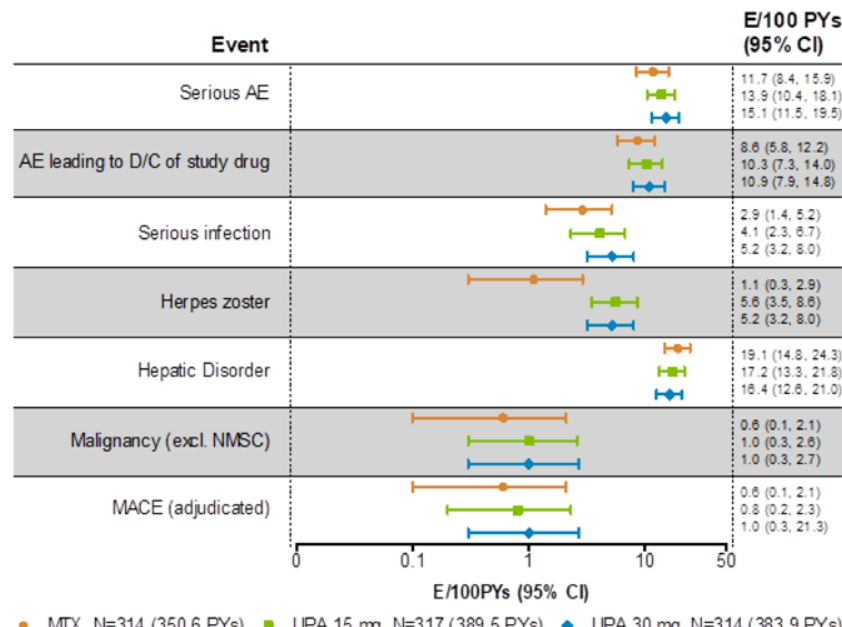

Figure. Treatment-emergent Adverse Events Through $\geq 72$ Weeks (E/100 PYs, 95\% Cl). 